\title{
On the Existence of Benthic Storms
}

Ronald Quirchmayr

To cite this article: Ronald Quirchmayr (2015) On the Existence of Benthic Storms, Journal of Nonlinear Mathematical Physics 22:4, 540-544, DOI:

https://doi.org/10.1080/14029251.2015.1113053

To link to this article: https://doi.org/10.1080/14029251.2015.1113053

Published online: 04 January 2021 


\title{
On the Existence of Benthic Storms
}

\author{
Ronald Quirchmayr \\ Faculty of Mathematics, University of Vienna, Oskar-Morgenstern-Platz 1 \\ 1090 Wien, Austria \\ ronald.quirchmayr@univie.ac.at
}

Received 11 September 2015

Accepted 12 October 2015

\begin{abstract}
We study a model for the wind-induced current field of the Pacific ocean in order to demonstrate that currents in the surface layer are carried down to the deepest regions above the abyssal sea floor, which indicates the existence of the phenomenon of comparably strong currents in bottom regions as a result of wind-stress forces at the surface, also known as benthic storms.
\end{abstract}

Keywords: equatorial flows; eddy viscosity; stratification.

2000 Mathematics Subject Classification: 86A05

\section{Introduction}

A longtime assumption in oceanography was that the water in the benthic boundary layer (BBL) above the abyssal sea floor, which develops as a result of friction at the bed, would be rather still. Former theory of the BBL with focus on mean-flow properties relied on models assuming horizontally homogeneous stationary currents (see $[3,8])$. The consideration of such models stemmed from the insufficient availability of empirical data for the hydrodynamics (but also the chemical and biological processes, etc. and their interactions) in the BBL, due to its inaccessibility in the open ocean. The perception changed fundamentally as a consequence of observations that have been made during long term projects such as the High Energy Benthic Boundary Layer Experiment (HEBBLE Program), which was primarily initiated for practical reasons such as the extraction of minerals from the see floor, anti submarine defense, navigation, the selection of waste burial sites, etc. It turned out that the BBL is not static at all. There are dramatic velocity increases in the benthic current, which occur periodically, the so-called benthic storms. Their importance springs from the fact that they stir up bottom sediments, which are then captured and transported over large distances by weaker but stable currents. Unlike the stationary weak currents, benthic storms seem to be controlled not only by forces resulting e.g. from thermohaline and tidal phenomena (also the Coriolis force plays a role in equatorial regions), but results from wind-stress at the surface layer, which is carried down to the BBL via mesoscale eddies having diameters up to $200 \mathrm{~km}$. This winddriven mechanism as origin of benthic storms particularly applies to equatorial regions in the Pacific (see [9]); there are regions where wind plays no or merely a minor role in the generation of benthic storms (see [11]).

The purpose of these notes is to prove - on the basis of a simple (static) model - that wind-stress forces at the surface indeed propagate down to the sea floor and thus may generate benthic storms. Let us point out, that such a basic model is not capable to describe the complex dynamics of the 
currents in the BBL. However it tells us that currents at the surface layer influence the flow in deep regions directly above the bottom.

\section{A note on currents in the equatorial region}

Our considerations rely on a linear eddy viscosity model in [4] for the wind-induced current field of the Pacific ocean in the equatorial region, where stratification is greater than anywhere else in the ocean (see [6]): a sharp thermocline separates a shallow water layer of warm water from a deep layer of colder water with higher density. The fluid domain, sketched in figure 1, consists of the subsurface layer

$$
U_{1}:=\left\{(x, z) \in \mathbb{R}^{2}:-h<z<0\right\}
$$

with constant water density $\rho$ between the water surface $\left\{(x, z) \in \mathbb{R}^{2}: z=0\right\}$ and the thermocline $\left\{(x, z) \in \mathbb{R}^{2}: z=-h\right\}$ (with $h>0$ ), and a deep layer

$$
U_{2}:=\left\{(x, z) \in \mathbb{R}^{2}:-d<z<-h\right\}
$$

beneath the thermocline and above the flat bottom $\left\{(x, z) \in \mathbb{R}^{2}: z=-d\right\}$ (with $d>h$ ) having a slightly higher density $\rho(1+r)$ for some fixed $r>0$.

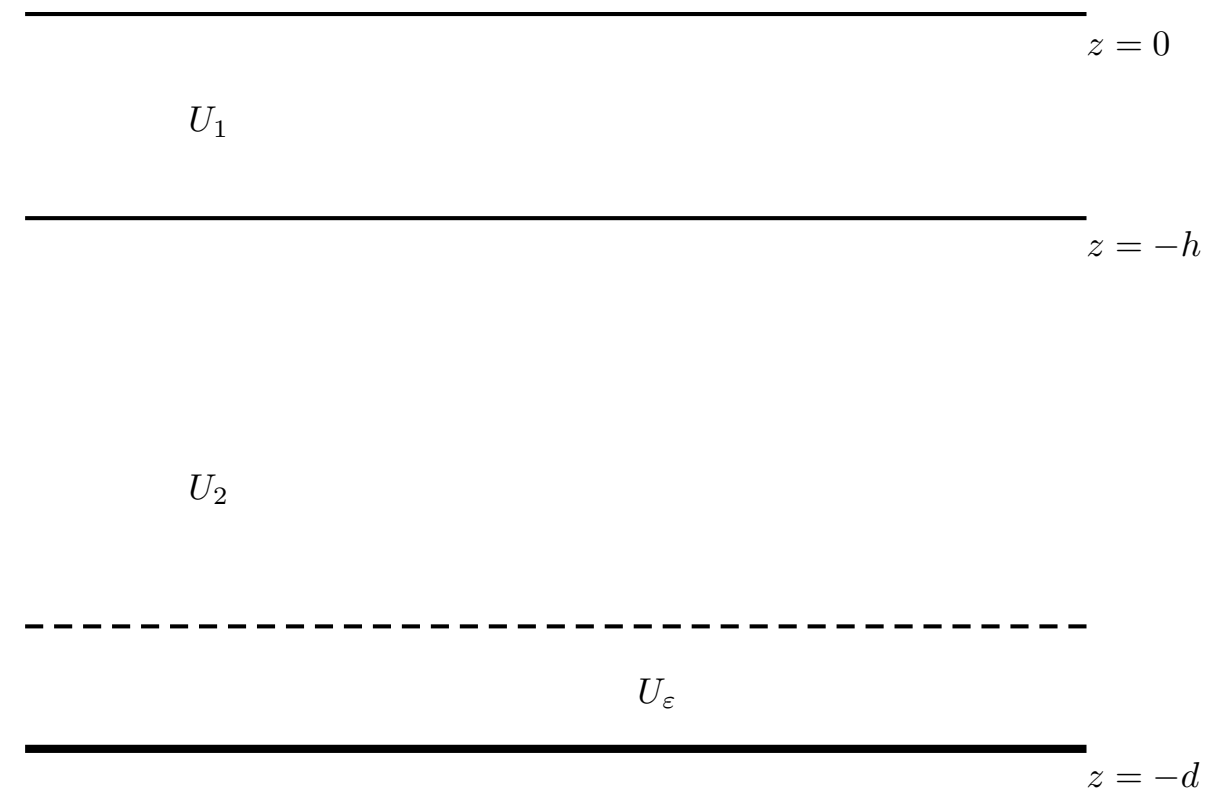

Fig. 1. The two layers are separated by the thermocline at a depth of roughly $120 \mathrm{~m}$. Most parts of the sea floor in the Pacific ocean are indeed flat, located at depths exceeding 3500m; so-called abyssal plains. 
We consider the linearized equations for a steady state flow with a vanishing vertical-fluidvelocity component in the $f$-plane approximation for both frictional layers. That is, presume

$$
\begin{aligned}
0 & =-\frac{1}{\rho} P_{x}+\left(v u_{z}\right)_{z}, \\
-2 \Omega u & =-\frac{1}{\rho} P_{z}-g, \\
u_{x} & =0
\end{aligned}
$$

throughout $U_{1}$, and accordingly we require

$$
\begin{aligned}
0 & =-\frac{1}{\rho(1+r)} P_{x}+\left(v u_{z}\right)_{z}, \\
-2 \Omega u & =-\frac{1}{\rho(1+r)} P_{z}-g \\
u_{x} & =0
\end{aligned}
$$

to hold within the deep layer $U_{2}$. The two unknowns - the velocity field $u$ and the pressure $P$ - are assumed to be smooth within $U_{1} \cup U_{2}$. The depth dependent viscosity parameter $v=v(z)$ is given. It is smooth throughout

$$
U:=U_{1} \cup U_{2} \cup\{z=-h\},
$$

positive and away from zero; in [5] it is suggested to take a suitable exponential decay to some small positive value as a good approximation for $v$. The constants $g$ and $\Omega$ denote the gravitational constant and the rotational speed of the earth around the polar axis toward the east.

In addition to equations (2.1)-(2.6) we consider the following boundary conditions. We impose a no-slip condition on the bottom, a vanishing vorticity of the velocity field on the thermocline and a constant atmospheric pressure on the surface. Furthermore we assume the velocity and the pressure to be continuous across the thermocline:

$$
\left\{\begin{array}{lll}
u=0 & \text { on } & \mathrm{z}=-\mathrm{d}, \\
u_{z}=0 & \text { on } & \mathrm{z}=-\mathrm{h}, \\
P=P_{\mathrm{atm}} & \text { on } & \mathrm{z}=0, \\
u, P \in \mathscr{C}(\bar{U}) . & &
\end{array}\right.
$$

The proposition below tells us that an absence of currents in regions above the sea floor would imply zero current up to the water surface. Let us denote such a bottom region by

$$
U_{\varepsilon}:=\{(x, z) \in \mathbb{R}:-d \leq z<-d+\varepsilon\}
$$

c.f. figure 1 .

Proposition 2.1. Assume that there is some region $U_{\varepsilon}$ above the bottom where the velocity field $u$ vanishes. Then $u$ is identically zero from the bottom to the surface.

Proof. We have that $\left(v u_{z}\right)_{z z}=0$ in $U_{1} \cup U_{2}$. In order to see this, we differentiate (2.2) with respect to $x$ first, and use (2.3) to infer that $P_{z x}=0$ in $U_{1}$. The claim follows by differentiating (2.1) with 
respect to $z$ and exploiting the smoothness of $P$ in $U_{1}$ :

$$
0=P_{z x}=P_{x z}=\left(v u_{z}\right)_{z z} \text { in } U_{1} .
$$

Analogously we get that $\left(v u_{z}\right)_{z z}=0$ in $U_{2}$. Therefore, $v u_{z}=A_{2} z+B$ for $A, B \in \mathbb{R}$, and by (2.8) we find that

$$
v u_{z}=A_{2}(z+h) \text { in } U_{2} .
$$

Since $u \equiv 0$ in $U_{\varepsilon}$, we find that $A_{2}=0$, hence $u_{z}=0$ in $U_{2}$ and from (2.7) and (2.10) we infer that

$$
u \equiv 0 \quad \text { in } \overline{U_{2}} .
$$

We may now infer from (2.4) and (2.5) that $P_{x} \equiv 0$ and $P_{z}=-g \rho(1+r)$, thus

$$
P(x, z)=-\rho(1+r) g z+C_{2}
$$

in $U_{2}$ for some constant of integration $C_{2} \in \mathbb{R}$.

Similarly we find that

$$
v u_{z}=A_{1}(z+h) \text { in } U_{1}
$$

for some $A_{1} \in \mathbb{R}$. Therefore (2.9), (2.10) and (2.12) imply that

$$
u(z)=\int_{-h}^{z} \frac{A_{1}(\xi+h)}{v(\xi)} \mathrm{d} \xi
$$

for $-h \leq z \leq 0$.

We obtain from (2.1) and (2.13) that $P_{x}=\rho A_{1}$ in $U_{1}$ and from (2.2) that $P_{z}=\rho(2 \Omega u-g)$ in $U_{1}$, thus

$$
P(x, z)=-\rho g z+2 \rho \Omega \int_{-h}^{z} u(\xi) \mathrm{d} \xi+\rho A_{1} x+C_{1}
$$

in $U_{1}$ for some constant of integration $C_{1} \in \mathbb{R}$. Due to (2.10), the following relation has to be fulfilled for all $x \in \mathbb{R}$ :

$$
\lim _{z \nearrow-h} P(x, z)=-\rho(1+r) g z+C_{2}=-\rho g z+\rho A_{1} x+C_{1}=\lim _{z \searrow-h} P(x, z) .
$$

This is only possible, if $A_{1}=0$ is satisfied. We deduce from (2.9) that $C_{2}=C_{1}-\rho r g h$, and $C_{1}=P_{\text {atm }}$. Hence the pressure field equals the hydrostatic pressure:

$$
P(x, z)= \begin{cases}P_{\mathrm{atm}}-\rho g z & \text { in } U_{1} \cup\{z=0\} \\ P_{\mathrm{atm}}-\rho(1+r) g z-\rho r g h & \text { in } U_{2} \cup\{z=-h\} \cup\{z=-d\} .\end{cases}
$$

Moreover, knowing that $A_{1}=0$, we obtain from (2.13) that $u_{z}=0$ throughout $U_{1}$, hence $u$ is constant within $U_{2}$. Therefore by (2.12) and the continuity assumption (2.10) we conclude that $u \equiv 0$ throughout $\bar{U}$.

Remark 2.1. Let us point out, that the region $U_{\varepsilon}$ does not particularly represent the BBL mentioned earlier in the introduction. It stands for some - theoretically arbitrary small - region directly above the bottom. 
The continuity assumption (2.10) can not be meaningfully strengthened in the sense of requiring $u$ and $P$ to be continuously differentiable or even smooth across the thermocline. We have seen in the proof of Proposition 2.1 that the hydrostatic pressure is only piecewise smooth; its $z$-derivative has a jump at the thermocline. Furthermore, requiring a smooth current $u$ throughout $U$, would trivialize the model. We would then already obtain that $\left(v u_{z}\right)_{z} \equiv 0$ in $U$ without imposing the additional condition of Proposition 2.1 (on some region $U_{\varepsilon}$ ). Hence we would get $u_{z} \equiv 0$ in $U$ by (2.8) and therefore the bottom condition (2.7) tells us that the only smooth solution of (2.1)-(2.3) and (2.4)(2.6) with boundary conditions (2.7)-(2.9) is the trivial one.

\section{Acknowledgment}

The author acknowledges support by the Austrian Science Fund (FWF), grant W1245.

\section{References}

[1] J. Y. Aller, Benthic community response to temporal and spatial gradients in physical disturbance within a deep-sea western boundary region, Deep-Sea Research I 44 (1997) 39-69.

[2] S. Beaulieu and R. Baldwin, Temporal variability in currents and the benthic boundary layer at an abyssal station off central California, Deep-Sea Research II 45 (1998) 587-615.

[3] B. P. Boudreau and B. B. Jørgensen, The Benthic Boundary Layer (Oxford University Press, 2001).

[4] A. Constantin and R. S. Johnson, The dynamics of waves interacting with the Equatorial Undercurrent, Geophysical and Astrophysical Fluid Dynamics 109 (2015) 311-358.

[5] M. F. Cronin and W. S. Kessler, Near-Surface Shear Flow in the Tropical Pacific Cold Tongue Front, Journal of Physical Oceanography 39 (2009) 1200-1215.

[6] A. V. Fedorov and J. N. Brown, Equatorial waves. In Encyclopedia of Ocean Sciences, edited by J. Steele, pp. 3679-3695, 2009 (Academic Press: New York).

[7] E. A. Kontar and A. V. Sokar, A benthic storm in the northeastern tropical Pacific over the fields of manganese nodules, Deep-Sea Research I 41 (1994) 1069-1089.

[8] E. A. Kontar and A. V. Sokar, On the benthic boundary layer's dynamics, Journal of Marine Systems 11 (1997) 369-385.

[9] T. Radziejewska, Meiobenthos in the Sub-equatorial Pacific Abyss (SpringerBriefs in Earth System Sciences, 2014).

[10] M. J. Richardson, G. L. Weatherly and W. D. Gardner, Benthic storms in the Argentine Basin, Deep-Sea Research II 40 (1993) 975-987.

[11] R. A. Woodgate and E. Fahrbach, Benthic storms in the Greenland sea, Deep-Sea Research I 46 (1999) 2109-2127. 